\title{
ESPRESSO: A High Resolution Spectrograph for the Combined Coudé Focus of the VLT
}

\author{
Luca Pasquini, A. Manescau, G. Avila, B. Delabre, H. Dekker, J. Liske, \\ S. D’Odorico, F. Pepe, M. Dessauges, C. Lovis, D. Megevand, D. Queloz, \\ S. Udry, S. Cristiani, P. Bonifacio, P. Dimarcantonio, V. D'Odorico, P. Molaro, \\ E. Vanzella, M. Viel, M. Haehnelt, B. Carswell, M. Murphy, R. Garcia-Lopez, \\ J.M. Herreros, J. Perez, M.R. Zapatero, R. Rebolo, G. Israelian, E. Martin, \\ F. Zerbi, P. Spanò, S. Levshakov, N. Santos and S. Zucker
}

\section{The Drivers}

In the frame of the call for proposal for the E-ELT instrumentation studies, the CODEX team carried out the feasibility study for a spectrograph for extremely stable Doppler measurements. The CODEX project and its scientific goals are described in [6]. During the development of this study, the CODEX team recognized that a CODEX-like instrument would be of high scientific interest also on the VLT. The ESPRESSO concept was born. The contribution by J. Liske in this volume highlights the direct links between the two instruments (see also [4]).

The ESPRESSO concept evolves from previous positives experiences at ESO, combining the stability of HARPS [3] with the efficiency of UVES and FEROS $[1,2]$. In summary, it is a high-efficiency, high-resolution, fiber-fed spectrograph of high mechanical and thermal stability using, if necessary, the simultaneous reference technique.

The first purpose of ESPRESSO is to be a competitive, innovative high-resolution spectrograph to fully exploit the VLT potentiality and to allow new science. ESPRESSO has indeed many very interesting applications, and several have been addressed in this conference by different speakers. The quest for enhanced radial velocity capabilities at the VLT for exo-planet search has been emphasized by Renzini, Bono, Queloz and Udry. P. Molaro discussed the relevance of investigating the variability of physical constants, and V. D' Odorico the results which can be obtained by studying the chemistry of the Intergalactic Medium. Finally, the detailed chemical analysis of stars will greatly benefit from ESPRESSO, as highlighted, for instance in the presentations by Bonifacio. Additional interesting topics are widely discussed in the proceedings of the "Precision Spectroscopy in Astrophysics" conference [7].

\footnotetext{
L. Pasquini $(\bowtie)$

ESO, Garching, Germany

e-mail:1pasquin@eso.org
} 
The second purpose of ESPRESSO is to gather fundamental experience for CODEX.

We finally find extremely exciting the possibility of using a $16 \mathrm{~m}$ equivalent telescope, in advance the E-ELT will be fully available to the community.

\section{From Requirements to Design}

The first challenge is to obtain the highest stability, while preserving an excellent efficiency. High spectrograph optomechanical stability is obtained through a controlled environment in vacuum and avoiding movable components. One critical item is the light input system, which must scramble the signal to ensure that the variability at the fiber input does not degrade the stability of the spectrograph, still keeping an excellent transmission. The requirement that ESPRESSO is kept in vacuum and thermally controlled implies containing the instrument volume and the optics size.

Obtaining the results from an integrated, system perspective: in order to obtain the demanding ESPRESSO performances, the whole chain must work, from object acquisition, to the data reduction and analysis. While a definitive answer will eventually come only from the use of the instrument, two main system tools have been adopted: first tool is the capitalization of the HARPS experience and its extension to the ESPRESSO requirements. Second tool is the extensive use of simulations generated to quantify the calibration requirements, the main requirements, the subsystem requirements.

A number of critical items have been identified, and they are addressed with dedicated R\&D efforts:

1. Efficiency: Improvements in efficiency with respect to HARPS include a shorter fiber length, a more efficient scrambling system and a two arm spectrograph design, with the use of two VPHs as crossdispersers.

2. Scrambling: This aspect differs between the 1-UT case and the 4-UTs case. In the 1-UT case the problem is analogue to the one of HARPS, but with 50 times more stringent requirements. In the 4-UT case there are 4 independent pupils; the scrambling shall therefore happen after combining the light from the sub-pupils, and to this scope 3 different systems are tested.

3. CCD temperature control: HARPS shows a correlation between detector temperature and radial velocity variations. A copy of the HARPS cryostat is studied, and design changes are made to improve this aspect. The construction of a prototype is planned.

4. Calibration System: A novel calibration system based on a laser frequency comb has been proposed and its feasibility is studied through a contract with MPQ. The final prototype is expected in 3 years from now (cf. Manescau et al., these proceedings).

5. Slanted VPHG: The slanted fringes VPHG is a potential innovative feature since, to the best of our knowledge, such a VPHG has never used in combination with a spectrograph, as crossdisperser and beam compressor. The use of slanted VPHs 
is not mandatory for ESPRESSO, but seems unavoidable for CODEX. We have therefore opted to proceed to the prototyping.

\section{The Design}

Following HARPS, ESPRESSO is designed with a dual fiber system, where the second fiber can be used either to record the sky or to monitor the spectrograph shifts by recording a simultaneous calibration source. It is actually one of the ESPRESSO aims to gather sufficient information for deciding if a simultaneous calibration fiber is required in CODEX, or if an operations scheme with interloped calibrations is acceptable for that instrument. ESPRESSO will sit in the Combined Coudé room, fed by a Coudé train which brings the light from the B Nasmyth platform to the room, where it is collected by the instrument acquisition and guide system into a fiber, which feeds the spectrograph. The spectrograph itself is contained in a vacuum tank enclosed in a thermally controlled room. No movable nor motorized functions are present inside the tank.

\subsection{The Coudé Train (see Avila et al.)}

Even if in the VLT provision for the Coudé optics is made, the design and hence the components of the train require to be developed. The ducts distance from each telescope to the combined focus is different for each UT; even if the concept for the Coudé train is the same for all UTs, each one will be different. The Coudé train has a FoV of 5 arcsec radius and it will be coated for an excellent response in the 300$750 \mathrm{~nm}$ wavelength range. Given the large distance traveled by the light in the duct, some induced seeing is expected at the fiber entrance, which will be compensated by a stabilization system in the fiber head. This component is critical, because the HARPS experience has shown as, even with a good scrambler, a movement of the source of 0.5 arcsec may induce a shift of up to a few $\mathrm{m} / \mathrm{sec}$.

\subsection{Calibration Unit (see Manescau et al.)}

The relevance of a novel, precise, predictable stable calibration system cannot be over-stressed and the characteristics of an ideal calibration source are described, for instance in [5]. The wavelength calibration unit for the spectrograph is based on Laser Frequency Comb system. Provision for the use of Th-Ar lamps and other lamps for flat field will be made. 


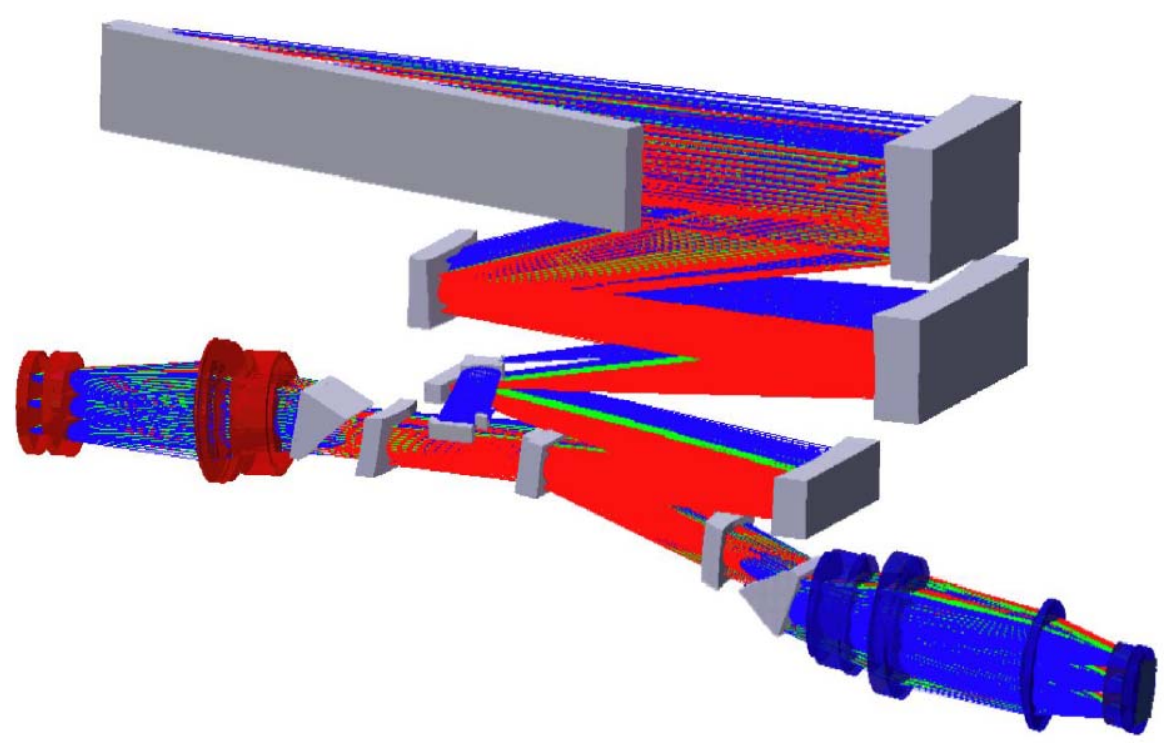

Fig. 1 The optical layout of ESPRESSO. The light, after injection form the fibers, passes through a pupil anamorphoser and the pupil is split. The two half-pupils are projected onto the echelle. The dichroic separates the blue and red arms, which are crossdispersed by VPH gratings. A colour version of this figure is available at dx.doi.org/10.1007/978-1-4020-9190-2_68

\subsection{Spectrograph Optics (see Spanò et al.)}

The basic concept for the spectrograph is a cross dispersed echelle with two arms. The echelle grating size is $1700 \times 200 \mathrm{~mm}$, consisting of a $4 \times 1$ mosaic of $408 \times$ $200 \mathrm{~mm}$ grating segments, or two UVES gratings. To limit the size of the echelle, pupil slicing is applied. The grating operates in near Littrow configuration.

The optical design makes use of anamorphism and pupil slicing. In this way a compact design is obtained, and a $20 \mathrm{~cm}$ optical beam and a $20 \times 160 \mathrm{~cm}$ echelle provides the resolving power of a un-sliced $40 \mathrm{~cm}$ beam spectrograph. The spectrograph optical design is given in Fig. 1.

\subsection{Mechanics \& Vacuum Vessel (see Zapatero and Osorio et al.)}

The spectrograph mechanics shall maintain the correct alignment and the configuration has been chosen to allow highest stability providing easy access for installation and maintenance. The whole spectrograph, including the detector head, is mounted on an optical bench and installed within a evacuated vacuum vessel with its temperature precisely controlled (few $\mathrm{mK}$ ). The vacuum tank will be hosted in a thermally controlled room in the Coudé lab. 
Table 1 Characteristics of the ESPRESSO design

\begin{tabular}{llll}
\hline Characteristic & Standard 1 UT & Faint object 4 UT & High efficiency 4 UT \\
\hline Wavelength range & $350-780 \mathrm{~nm}$ & $350-780 \mathrm{~nm}$ & $350-780 \mathrm{~nm}$ \\
Resolving power & 160000 & 40000 & 80000 \\
Sampling (average) & 4 pixels & 16 pixels & 8 pixels \\
Spatial pixels & 24 & 24 & 48 \\
Simultaneous calibration & YES & NO & YES \\
Sky subtraction & YES (either SimCal) & YES & YES (either SimCal) \\
\hline
\end{tabular}

\subsection{Instrument Control and Software (see Megevand et al.)}

The instrument control hardware consist in a number of LCUs and the different controllers to control the instrument functions. The Data Reduction Software will transform raw frames into clean, extracted, flat fielded, wavelength calibrated spectra. The main difference between the ESPRESSO DRS and the standard ESO pipelines is that it shall deliver the best science quality data on-line. Data Analysis SW is that part of the science SW which is non-common to the various scientific domains and requires specific tools and focus. The Data-Analysis SW is preferably automatic. For all tools the input is an extracted, wavelength-calibrated spectrum delivered by the DRS, and its output are scientific observables. We distinguish following domains for which different data-analysis tools/functions are required:

- Analysis of non-stellar spectra, low SNR science.

- Correlation, RVs, bi-sector analysis.

- General (stellar) spectroscopy.

- Package of other RV extraction methods.

\section{Performances}

ESPRESSO is proposed to have three operating modes, one with 1-UT and two with 4-UTs. The summary of the characteristics is given in Table 1.

\section{References}

1. H. Dekker, S. D’Odorico et al., SPIE 4008, 534 (2000)

2. A. Kaufer, L. Pasquini, SPIE 3355, 844 (1998)

3. M. Mayor, F. Pepe et al., Messenger 114, 20 (2003)

4. J. Liske, A. Grazian, E. Vanzella et al., 2008MNRAS. tmp..460L, in press

5. M. Murphy, T. Udem et al., Mon. Not. R. Astron. Soc. 379, 1407 (2007)

6. L. Pasquini, S. Cristiani et al., Messenger 122, 10 (2005)

7. N. Santos, L. Pasquini, A. Correia, M. Romaniello, Precision Spectroscopy in Astrophysics (Springer, Berlin, 2007) 